\title{
Approximate Controllability for Systems Governed by Nonlinear Volterra Type Equations
}

\section{Tran Dinh Ke, Valeri Obukhovskii, Ngai-Ching Wong \& Jen-Chih Yao}

Differential Equations and Dynamical

\section{Systems}

International Journal for Theory, Real

World Modelling and Simulations

ISSN 0971-3514

Differ Equ Dyn Syst

DOI 10.1007/s12591-011-0101-7
ISSN 0971-3514

Volume $16 \cdot$ Numbers $1-2 \cdot$ January-April 2008

Differential

Equations and

Dynamical

Systems

An Official Publication of

Foundation for

Scientific Research and

Technological Innovation

International Journal for Theory, Real World Modelling and Simulations

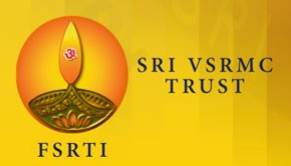

Springer

算 Springer 
Your article is protected by copyright and all rights are held exclusively by Foundation for Scientific Research and Technological Innovation. This e-offprint is for personal use only and shall not be self-archived in electronic repositories. If you wish to selfarchive your work, please use the accepted author's version for posting to your own website or your institution's repository. You may further deposit the accepted author's version on a funder's repository at a funder's request, provided it is not made publicly available until 12 months after publication. 


\title{
Approximate Controllability for Systems Governed by Nonlinear Volterra Type Equations
}

\author{
Tran Dinh Ke • Valeri Obukhovskii • \\ Ngai-Ching Wong • Jen-Chih Yao
}

(C) Foundation for Scientific Research and Technological Innovation 2011

\begin{abstract}
We study the control systems governed by abstract Volterra equations without uniqueness in a Banach space. By using the technique of the theory of condensing maps and multivalued analysis tools, we obtain the existence result, investigate the topological structure of the solution set, and prove the invariance of a reachability set of the control system under nonlinear perturbations. Examples concerning fractional order differential equations and first order evolution equations with multiple delays are proposed to demonstrate the applications to approximate controllability results.
\end{abstract}

Keywords Reachability set · Abstract Volterra equation - Fixed point · Non-convex valued multimap · Measure of non-compactness - Exact controllability · Approximate controllability $\cdot$ AR-space $\cdot$ ANR-space $\cdot R_{\delta}$-map $\cdot$ Fractional order control problem . Multiple delays

Mathematics Subject Classification (2010) 93B05 - 93B03 - 93B28 .

$93 \mathrm{C} 10 \cdot 93 \mathrm{C} 25 \cdot 47 \mathrm{H} 04 \cdot 47 \mathrm{H} 08 \cdot 47 \mathrm{H} 10$

T. D. Ke

Department of Mathematics, Hanoi National University of Education,

136 Xuan Thuy, Cau Giay, Hanoi, Vietnam

e-mail: ketd@hn.vnn.vn

V. Obukhovskii $(\varangle)$

Faculty of Physics and Mathematics, Voronezh State Pedagogical University, 394043 Voronezh, Russia e-mail: valerio-ob2000@mail.ru

N.-C. Wong · J.-C. Yao

Department of Applied Mathematics, National Sun Yat-sen University, 804 Kaohsiung, Taiwan, ROC e-mail: wong@math.nsysu.edu.tw

J.-C. Yao

Center for General Education, Kaohsiung Medical University, 807 Kaohsiung, Taiwan, ROC

e-mail: yaojc@cc.kmu.edu.tw 


\section{Introduction}

Let $X$ and $V$ be Banach spaces. We consider the following control system

$$
x(t)=x^{*}(t)+\int_{0}^{t} \Phi(t, s)[f(s, x(s))+B u(s)] d s, t \in[0, T],
$$

where the state function $x$ takes values in $X$, the control $u$ takes values in $V, f:[0, T] \times$ $X \rightarrow X$ is a nonlinear map and $B: V \rightarrow X$ is a bounded linear operator. Here also $x^{*} \in C([0, T] ; X)$ is a given function and $\Phi(t, s)$ is a bounded linear operator in $X$ for each $t, s \in[0, T], t \geq s$.

It is well-known that system (1.1) arises from many classes of Cauchy problems for differential equations, in which $\Phi$ usually stands for a semigroup or an evolution process. In many papers the exact controllability for nonlinear systems was considered with the suggestion that the linear controllability operator $\mathcal{W} u=\int_{0}^{T} \Phi(T, s) B u(s) d s$ is pseudo-invertible, i.e., $\mathcal{W}$ must be surjective. See, e.g., [2,23] and other related works. However, as it was pointed out in [25,26], this assumption on $\mathcal{W}$ can not be satisfied in the cases when $X$ is an infinite-dimensional space and $\Phi$ (or $B$ ) is a compact operator. Therefore, the concept of exact controllability is too strong and in these cases, the notion of approximate controllability is more useful.

In [24] the controllability problem for system (1.1) was treated by proving that the reachability set is invariant under nonlinear perturbations. By using this approach, a number of works were carried out for a wide class of semilinear control systems, see [16-18] and references therein. It should be noted however that in these works the authors assumed the uniqueness of a solution and, as the consequence, they obtained the fact that the solution map $W u:=x(\cdot)$ is a single-valued and compact map. The uniqueness can be provided by the assumption that the nonlinearity $f$ is a Lipschitz function. In order to fulfill the compactness supposition, the compactness of $\Phi$ or some restricting hypotheses on $f$ were imposed. We refer the readers to [24] for details.

In the present work, by using the concept of a measure of non-compactness (MNC), we deal with a more general class of nonlinearities $f$ which does not guarantee the uniqueness of a solution for Eq. 1.1. However, by using the fixed point approach for multivalued maps, we can apply the similar arguments as in [24] to show that the reachability set for control system (1.1) is the same as that of the corresponding linear problem (when $f=0$ ). The lack of uniqueness prevents us from using the well-known tools such as Banach and Schauder fixed point theorems to show the invariance of reachable set for (1.1) as in [24]. This difficulty leads us to study the structure of the solution set for (1.1), before applying a fixed point theory for non-convex valued multimaps. It is worth noting that in our problem, $\Phi(t, s)$ does not need to be a semigroup neither an evolution operator, so we can impose general conditions on $\Phi$ and provide the applications of abstract results to various problems, examples of which are presented in the last section.

This article is organized in the following way. In the next section, we prove the existence result for (1.1) under suitable assumptions on $\Phi$ and $f$. In particular, it is assumed that the nonlinearity $f$ satisfies a regularity condition expressed in terms of a MNC. This allows us to employ the technique of the fixed point theory for condensing maps to solve the problem. It should be noted that such an approach was developed in [12] and its application to a controllability problem can be found in [20]. In "Topological Structure of the Solution Set" section, we prove some properties related to the topological structure of the solution set, which allows 
to deduce a fixed point result in "Invariance of the Reachability Set" section and opens the possibility to prove the invariance of reachability set for our problem under nonlinear perturbations. In the last section we consider the applications to approximate controllability for two classes of controlled problems: the first problem relates to a fractional order differential equation and the second one concerns a first order evolution equation with multiple delays.

\section{Existence Result}

Let us recall some definitions and results which will be used in the sequel.

Definition 2.1 Let $\mathcal{E}$ be a Banach space, $\mathcal{P}(\mathcal{E})$ denote the collection of all nonempty subsets of $\mathcal{E}$, and $(\mathcal{A}, \geqslant)$ a partially ordered set. A function $\beta: \mathcal{P}(\mathcal{E}) \rightarrow \mathcal{A}$ is called a MNC in $\mathcal{E}$ if

$$
\beta(\overline{c o} \Omega)=\beta(\Omega) \text { for every } \Omega \in \mathcal{P}(\mathcal{E}),
$$

where $\overline{c o} \Omega$ is the closure of convex hull of $\Omega$. A MNC $\beta$ is called

(i) monotone, if for each $\Omega_{0}, \Omega_{1} \in \mathcal{P}(\mathcal{E})$ such that $\Omega_{0} \subset \Omega_{1}$, we have $\beta\left(\Omega_{0}\right) \leqslant \beta\left(\Omega_{1}\right)$

(ii) nonsingular, if $\beta(\{a\} \cup \Omega)=\beta(\Omega)$ for any $a \in \mathcal{E}, \Omega \in \mathcal{P}(\mathcal{E})$;

(iii) invariant with respect to the union with a compact set, if $\beta(K \cup \Omega)=\beta(\Omega)$ for every relatively compact set $K \subset \mathcal{E}$ and $\Omega \in \mathcal{P}(\mathcal{E})$;

If $\mathcal{A}$ is a cone in a normed space, we say that $\beta$ is

(iv) algebraically semi-additive, if $\beta\left(\Omega_{0}+\Omega_{1}\right) \leqslant \beta\left(\Omega_{0}\right)+\beta\left(\Omega_{1}\right)$ for any $\Omega_{0}, \Omega_{1} \in \mathcal{P}(\mathcal{E})$;

(v) regular, if $\beta(\Omega)=0$ is equivalent to the relative compactness of $\Omega$.

An important example of MNC, satisfying all above properties, is the Hausdorff MNC:

$$
\chi(\Omega)=\inf \{\varepsilon: \Omega \text { has a finite } \varepsilon \text {-net }\} .
$$

Another examples of MNCs on the space $C([0, T] ; X)$ of continuous functions on interval $[0, T]$ taking values in a Banach space $X$ are the following:

(i) the damped modulus of fiber non-compactness

$$
\gamma(\Omega)=\sup _{t \in[0, T]} e^{-L t} \chi(\Omega(t))
$$

where $L$ is a nonnegative constant, $\chi$ is the Hausdorff MNC on $X$ and $\Omega(t)=\{y(t)$ : $y \in \Omega\}$;

(ii) the modulus of equicontinuity

$$
\bmod _{C}(\Omega)=\lim _{\delta \rightarrow 0} \sup _{y \in \Omega} \max _{\left|t_{1}-t_{2}\right|<\delta}\left\|y\left(t_{1}\right)-y\left(t_{2}\right)\right\| .
$$

As it was indicated in [12], these MNCs satisfy all the above mentioned properties except the regularity.

Definition 2.2 A continuous map $\mathcal{F}: X \subset \mathcal{E} \rightarrow \mathcal{E}$ is said to be condensing with respect to a MNC $\beta$ ( $\beta$-condensing) if for every bounded set $\Omega \subset X$ that is not relatively compact, we have

$$
\beta(\mathcal{F}(\Omega)) \nsupseteq \beta(\Omega) .
$$


The application of the topological degree theory for condensing maps (see [12]) implies the following fixed point principle.

Theorem 2.1 Let $\mathcal{V} \subset \mathcal{E}$ be a bounded open neighborhood of zero and $\mathcal{F}: \overline{\mathcal{V}} \rightarrow \mathcal{E}$ a $\beta$-condensing map with respect to a monotone nonsingular $M N C \beta$ in $\mathcal{E}$. If $\mathcal{F}$ satisfies the boundary condition

$$
x \neq \lambda \mathcal{F}(x)
$$

for all $x \in \partial \mathcal{V}$ and $0<\lambda \leqslant 1$ then the fixed point set $F i x(\mathcal{F})=\{x: x=\mathcal{F}(x)\}$ is nonempty and compact.

Now we will describe conditions which will be imposed on maps in (1.1).

Let $X$ be a Banach space, $p \geqslant 1$ be given and $q$ the conjugate of $p$ (i.e., $\frac{1}{p}+\frac{1}{q}=1$ and $q=+\infty$ if $p=1$ ). We assume that

$\left(\Phi_{1}\right)$ there exists a function $\rho \in L^{q}(0, T)$ such that $\|\Phi(t, s)\|_{X \rightarrow X} \leqslant \rho(t-s)$ for all $t, s \in[0, T], t \geq s$

$\left(\Phi_{2}\right)\|\Phi(t, s)-\Phi(r, s)\|_{X \rightarrow X} \leqslant \epsilon$ for $0 \leqslant s \leqslant r-\epsilon, r<t=r+h \leqslant T$ with $\epsilon=\epsilon(h) \rightarrow 0$ as $h \rightarrow 0$.

We now suppose that the nonlinearity $f$ satisfies the following conditions:

(F1) the map $f:[0, T] \times X \rightarrow X$ is continuous;

(F2) there exists a function $\mu \in L^{p}(0, T)$ such that

$$
\|f(t, \eta)\|_{X} \leqslant \mu(t)\left(1+\|\eta\|_{X}\right)
$$

for all $t \in[0, T]$ and $\eta \in X$;

(F3) there exists a function $k:[0, T] \times[0, T] \rightarrow \mathbb{R}^{+}$such that $k(t, \cdot) \in L^{1}(0, t)$ for each $t \in[0, T]$ and

$$
\chi(\Phi(t, s) f(s, \Omega)) \leqslant k(t, s) \chi(\Omega)
$$

for a.e. $s \in[0, t]$ and for all bounded set $\Omega \subset X$, where $\chi$ is the Hausdorff MNC in $X$.

Remark 2.1 1. If $X$ is a finite-dimensional space, one can exclude the hypothesis (F3) since it can be deduced from (F2).

2. It is known that (see e.g., [12]) condition (F3) is fulfilled if

$$
f(s, \eta)=f_{1}(s, \eta)+f_{2}(s, \eta)
$$

where $f_{1}$ is Lipschitzian with respect to the second argument:

$$
\left\|f_{1}(s, \xi)-f_{1}(s, \eta)\right\|_{X} \leqslant h(s)\|\xi-\eta\|_{X}
$$

for some $h \in L^{p}(0, T)$ and $f_{2}$ is compact in second argument, i.e., for each $s \in[0, T]$ and bounded $\Omega \subset X$, the set $f_{2}(s, \Omega)$ is relatively compact in $X$. In this case, one can take $k(t, s)=\rho(t-s) h(s)$.

Denote by $\mathbf{S}: L^{p}(0, T ; X) \rightarrow C([0, T] ; E)$ the operator given by

$$
(\mathbf{S} g)(t):=\int_{0}^{t} \Phi(t, s) g(s) d s .
$$

The following result was proved in [24, Lemma 1]. 
Lemma 2.2 ([24]) Assume that $\Phi$ satisfies $\left(\Phi_{1}\right)-\left(\Phi_{2}\right)$. Then the operator $\mathbf{S}$ defined in (2.3) sends each bounded set to equicontinuous one.

Consider an abstract operator $\mathcal{L}: L^{1}(0, T ; X) \rightarrow C([0, T] ; X)$ satisfying the following conditions:

$(\mathcal{L} 1)$ there exists a constant $C>0$ such that

$$
\|\mathcal{L}(f)(t)-\mathcal{L}(g)(t)\|_{X} \leq C \int_{0}^{t}\|f(s)-g(s)\|_{X} d s,
$$

for all $f, g \in L^{1}([0, T] ; X), t \in[0, T]$;

$(\mathcal{L} 2)$ for each compact set $K \subset X$ and sequence $\left\{f_{n}\right\} \subset L^{1}([0, T] ; X)$ such that $\left\{f_{n}(t)\right\}$ $\subset K$ for a.e. $t \in[0, T]$, the weak convergence $f_{n} \rightarrow f_{0}$ implies $\mathcal{L}\left(f_{n}\right) \rightarrow \mathcal{L}\left(f_{0}\right)$ strongly in $C([0, T] ; X)$.

As it was mentioned in [12, Remark 4.2.3], the Cauchy operator

$$
\mathcal{G}_{I}(f)(t)=\int_{0}^{t} f(s) d s
$$

satisfies $(\mathcal{L} 1)-(\mathcal{L} 2)$ with $C=1$.

Notice the following assertion providing us with a basic MNC-estimate.

Lemma 2.3 ([12]) Let $\mathcal{L}$ satisfy $(\mathcal{L} 1)-(\mathcal{L} 2)$ and a sequence $\left\{\xi_{n}\right\} \subset L^{1}([0, T] ; X)$ be integrably bounded, i.e.,

$$
\left\|\xi_{n}(t)\right\| \leq v(t) \text { for a.e. } t \in[0, T],
$$

where $v \in L^{1}(0, T)$. Assume that there exists $q \in L^{1}([0, T])$ such that

$$
\chi\left(\left\{\xi_{n}(t)\right\}\right) \leq q(t) \text { for a.e. } t \in[0, T] .
$$

Then

$$
\chi\left(\left\{\mathcal{L}\left(\xi_{n}\right)(t)\right\}\right) \leq 2 C \int_{0}^{t} q(s) d s
$$

for each $t \in[0, T]$.

For a given $u \in L^{p}(0, T ; V)$, consider the operator

$$
\begin{aligned}
& \mathcal{F}^{u}: \quad C([0, T] ; X) \rightarrow C([0, T] ; X), \\
& \mathcal{F}^{u}(x)(t)=x^{*}(t)+\int_{0}^{t} \Phi(t, s)[f(s, x(s))+B u(s)] d s .
\end{aligned}
$$

It is easy to check that $\mathcal{F}^{u}$ is continuous. Moreover, a function $x \in C([0, T] ; X)$ is a solution of problem (1.1) if and only if $x$ is a fixed point of $\mathcal{F}^{u}$.

Consider now the function

$$
\begin{aligned}
& v: \mathcal{P}(C([0, T] ; X)) \rightarrow \overline{\mathbb{R}}_{+}^{2}, \\
& v(\Omega)=\max _{D \in \Delta(\Omega)}\left(\gamma(D), \bmod _{C}(D)\right),
\end{aligned}
$$


where $\overline{\mathbb{R}}_{+}=\mathbb{R}_{+} \cup\{+\infty\}$, the MNCs $\gamma$ and $\bmod _{C}$ are defined in (2.1) and (2.2) respectively, $\Delta(\Omega)$ is the collection of all countable subsets of $\Omega$ and the maximum is taken in the sense of the partial order in the cone $\overline{\mathbb{R}}_{+}^{2}$. By the same arguments as in [12], one can see that $v$ is well defined. That is, the maximum is archieved in $\Delta(\Omega)$ and $v$ is a MNC in the space $C([0, T] ; X)$, which satisfies all properties in Definition 2.1 (see [12, Example 2.1.3] for details).

Let us now choose $L>0$ in the definition of $\gamma$ such that

$$
\ell=\sup _{t \in[0, T]} 2 \int_{0}^{t} e^{-L(t-s)} k(t, s) d s<1,
$$

where $k$ is the function from condition $(\mathrm{F} 3)$.

Theorem 2.4 Let $\Phi$ satisfy $\left(\Phi_{1}\right)-\left(\Phi_{2}\right)$ and $f$ have properties $(\mathrm{F} 1)-(\mathrm{F} 3)$. Then for each $u \in L^{p}(0, T ; V), \mathcal{F}^{u}$ is $v$-condensing.

Proof Let $\Omega \subset C([0, T] ; X)$ be a bounded set such that

$$
v\left(\mathcal{F}^{u}(\Omega)\right) \geqslant v(\Omega) .
$$

We will show that $\Omega$ is relatively compact in $C([0, T] ; X)$. By the definition of $v$, there exists a sequence $\left\{z_{n}\right\} \subset \mathcal{F}^{u}(\Omega)$ such that

$$
v\left(\mathcal{F}^{u}(\Omega)\right)=\left(\gamma\left(\left\{z_{n}\right\}\right), \bmod _{C}\left(\left\{z_{n}\right\}\right)\right) .
$$

Following the construction of $\mathcal{F}^{u}$, one can take a sequence $\left\{x_{n}\right\} \subset \Omega$ and the sequence $\left\{f_{n}\right\} \subset L^{p}(0, T ; X)$ such that

$$
f_{n}(t)=f\left(t, x_{n}(t)\right)+B u(t)
$$

and

$$
z_{n}=x^{*}+\mathbf{S} f_{n}
$$

i.e.,

$$
z_{n}(t)=x^{*}(t)+\int_{0}^{t} \Phi(t, s) f_{n}(s) d s
$$

Using assumption (F3), we have

$$
\begin{aligned}
\chi\left(\left\{\Phi(t, s) f_{n}(s)\right\}\right) & =\chi\left(\Phi(t, s) f\left(s,\left\{x_{n}(s)\right\}\right)\right) \\
& \leqslant k(t, s) \chi\left(\left\{x_{n}(s)\right\}\right) \\
& \leqslant k(t, s) e^{L s} \sup _{s \in[0, T]} e^{-L s} \chi\left(\left\{x_{n}(s)\right\}\right)=k(t, s) e^{L s} \gamma\left(\left\{x_{n}\right\}\right),
\end{aligned}
$$

for all $t \in[0, T], s \leqslant t$. Then applying Lemma 2.3 with $\mathcal{L}=\mathcal{G}_{I}$, we obtain

$$
\chi\left(\left\{\mathbf{S} f_{n}(t)\right\}\right)=\chi\left(\left\{\int_{0}^{t} \Phi(t, s) f_{n}(s) d s\right\}\right) \leqslant 2\left(\int_{0}^{t} e^{L s} k(t, s) d s\right) \gamma\left(\left\{x_{n}\right\}\right) .
$$


This implies

$$
e^{-L t} \chi\left(\left\{\mathbf{S} f_{n}(t)\right\}\right) \leqslant 2\left(\int_{0}^{t} e^{-L(t-s)} k(t, s) d s\right) \gamma\left(\left\{x_{n}\right\}\right) .
$$

Taking (2.7) and (2.10) into account, we arrive at

$$
\gamma\left(\left\{z_{n}\right\}\right) \leqslant \ell \gamma\left(\left\{x_{n}\right\}\right) .
$$

Combining the last inequality with (2.8), we have

$$
\gamma\left(\left\{x_{n}\right\}\right) \leqslant \ell \gamma\left(\left\{x_{n}\right\}\right)
$$

and therefore

$$
\gamma\left(\left\{x_{n}\right\}\right)=0
$$

by choosing $\ell$ in (2.7). Hence by (2.13) we get

$$
\gamma\left(\left\{z_{n}\right\}\right)=0 .
$$

In addition, from (F2) and (2.9) we conclude that $\left\{f_{n}\right\}$ is a bounded sequence in $L^{p}(0, T ; X)$. Then Lemma 2.2 ensures that $\left\{\mathbf{S} f_{n}\right\}$ is equicontinuous in $C([0, T] ; X)$. Thus, $\bmod _{C}\left(\left\{z_{n}\right\}\right)=$ $\bmod _{C}\left(\left\{\mathbf{S} f_{n}\right\}\right)=0$. Finally,

$$
v(\Omega)=(0,0)
$$

and therefore, by the regularity of $v$, the set $\Omega$ is relatively compact.

In the sequel we will need the following version of the Gronwall-Bellman Inequality (see, e.g., [22]).

Lemma 2.5 Assume that $f(t), g(t)$ and $y(t)$ are non-negative integrable functions in $[0, T]$ satisfying the integral inequality

$$
y(t) \leqslant g(t)+\int_{0}^{t} f(s) y(s) d s, t \in[0, T] .
$$

Then we have

$$
y(t) \leqslant g(t)+\int_{0}^{t} \exp \left\{\int_{s}^{t} f(\theta) d \theta\right\} f(s) g(s) d s, t \in[0, T] .
$$

Now we are in position to present the main result of this section.

Theorem 2.6 Suppose that $\left(\Phi_{1}\right)-\left(\Phi_{2}\right)$ hold and $f$ satisfies $(\mathrm{F} 1)-(\mathrm{F} 3)$. Then for each $u \in$ $L^{p}(0, T ; V)$, problem $(1.1)$ has at least one solution in $C([0, T] ; X)$.

Proof In order to apply Theorem 2.1, we will prove that if $x=\lambda \mathcal{F}^{u}(x)$ for some $0<\lambda \leqslant 1$ then $x$ must belong to a priori bounded set in $C([0, T] ; X)$. Indeed, let $x=\lambda \mathcal{F}^{u}(x)$, i.e.,

$$
x(t)=\lambda\left(x^{*}(t)+\int_{0}^{t} \Phi(t, s)(f(s, x(s))+B u(s)) d s\right) .
$$


Then the application of the Hölder inequality and property (F2) yields the following estimates

$$
\begin{aligned}
\|x(t)\|_{X} & \leqslant C_{0}+C_{\Phi}\left(\int_{0}^{t}\|f(s, x(s))\|_{X}^{p}\right)^{\frac{1}{p}}+C_{\Phi}\|B u\|_{L^{p}(0, T ; X)} \\
& \leqslant C_{0}+2 C_{\Phi}\left(\int_{0}^{t}|\mu(s)|^{p}\left(1+\|x(s)\|_{X}^{p}\right)\right)^{\frac{1}{p}}+C_{\Phi}\|B u\|_{L^{p}(0, T ; X)},
\end{aligned}
$$

where $C_{0}=\left\|x^{*}\right\|_{C([0, T] ; X)}$ and

$$
C_{\Phi}= \begin{cases}\left(\int_{0}^{T}|\rho(s)|^{q} d s\right)^{\frac{1}{q}}, & \text { if } q<+\infty, \\ \sup _{t \in[0, T]} \rho(t), & \text { if } q=+\infty .\end{cases}
$$

This implies

$$
\|x(t)\|_{X}^{p} \leqslant 2^{p-1}\left(C_{0}^{p}+2^{p} C_{\Phi}^{p} C_{\mu}^{p}+C_{\Phi}^{p}\|B u\|_{L^{p}(0, T ; X)}^{p}+\int_{0}^{t}|\mu(s)|^{p}\|x(s)\|_{X}^{p} d s\right)
$$

where $C_{\mu}=\|\mu\|_{L^{p}(0, T)}$. Now applying the Bellman-Gronwall inequality given in Lemma 2.5 we get

$$
\|x(t)\|_{X}^{p} \leqslant g_{0}\left(1+2^{p-1} C_{\mu}^{p} e^{2^{p-1} C_{\mu}^{p}}\right)
$$

where $g_{0}=2^{p-1}\left(C_{0}^{p}+2^{p} C_{\Phi}^{p} C_{\mu}^{p}+C_{\Phi}^{p}\|B u\|_{L^{p}(0, T ; X)}^{p}\right)$. Then we can take the ball $B_{R}$ centered at origin with radius $R>g_{0}^{\frac{1}{p}}\left(1+2^{p-1} C_{\mu}^{p} e^{2^{p-1}} C_{\mu}^{p}\right)^{\frac{1}{p}}$ and apply Theorem 2.1 to the restriction of $\mathcal{F}^{u}$ onto $B_{R}$.

\section{Topological Structure of the Solution Set}

Let $Y$ and $Z$ be metric spaces. A multi-valued map (multimap) $G: Y \rightarrow \mathcal{P}(Z)$ is said to be: (i) upper semi-continuous (u.s.c.) if the set

$$
G_{+}^{-1}(V)=\{y \in Y: G(y) \subset V\}
$$

is open for any open set $V \subset Z$; (ii) closed if its graph $\Gamma_{G} \subset Y \times Z$,

$$
\Gamma_{G}=\{(y, z): z \in G(y)\}
$$

is a closed subset of $Y \times Z$.

The multimap $G$ is called quasi-compact if its restriction to any compact set is compact. The following statement gives a sufficient condition for upper semi-continuity.

Lemma 3.1 ([12]) Let $Y$ and $Z$ be metric spaces and $G: Y \rightarrow \mathcal{P}(Z)$ a closed quasi-compact multimap with compact values. Then $G$ is u.s.c. 
Consider the solution multimap

$$
\begin{aligned}
& W: \quad L^{p}(0, T ; V) \rightarrow \mathcal{P}(C([0, T] ; X)) \\
& W(u)=\left\{x: x=\mathcal{F}^{u}(x)\right\}
\end{aligned}
$$

We need an additional assumption on $\Phi$ :

$\left(\Phi_{3}\right) \Phi(t, s)$ is compact for all $t>s$.

Lemma 3.2 Under assumptions $\left(\Phi_{1}\right)-\left(\Phi_{3}\right)$ and $(\mathrm{F} 1)-(\mathrm{F} 2)$, the solution multimap $W$ defined by (3.1) is completely continuous, i.e., it is u.s.c. and sends each bounded set to a relatively compact one.

Proof Let $\mathcal{Q} \subset L^{p}(0, T ; V)$ be a bounded set. We prove that $W(\mathcal{Q})$ is relatively compact in $C([0, T] ; X)$. Suppose that $\left\{x_{n}\right\} \subset W(\mathcal{Q})$. Then there exists a sequence $\left\{u_{n}\right\} \subset \mathcal{Q}$ such that

$$
x_{n}(t)=x^{*}(t)+\int_{0}^{t} \Phi(t, s)\left[f\left(s, x_{n}(s)\right)+B u_{n}(s)\right] d s .
$$

Equivalently, we can write

$$
x_{n}=x^{*}+\mathbf{S}\left(f_{n}+B u_{n}\right),
$$

where $f_{n}(t)=f\left(t, x_{n}(t)\right)$. We observe that $\left\{B u_{n}\right\}$ is a bounded set in $L^{p}(0, T ; X)$ since $B$ is a bounded linear operator. This implies, by property $\left(\Phi_{3}\right)$, that $\left\{\mathbf{S}\left(B u_{n}\right)(t)\right\}$ is compact in $X$ for each $t \in[0, T]$ and we obtain from (3.3) that

$$
\chi\left(\left\{x_{n}(t)\right\}\right)=\chi\left(\left\{\mathbf{S} f_{n}(t)\right\}\right)=0
$$

for all $t \in[0, T]$. On the other hand, by using similar arguments as in the proof of Theorem 2.6, we can obtain that $\left\{x_{n}\right\}$ is a bounded sequence in $C([0, T] ; X)$. This implies that $\left\{f_{n}\right\}$ is also bounded in $L^{p}(0, T ; X)$ and Lemma 2.2 ensures that $\left\{\mathbf{S}\left(f_{n}+B u_{n}\right)\right\}$ is equicontinuous. In view of (3.3), we conclude that $\left\{x_{n}\right\}$ is also equicontinuous. Therefore $\left\{x_{n}\right\}$ is relatively compact by the Arzela-Ascoli theorem.

In order to prove that $W$ is u.s.c., it remains to show, according to Lemma 3.1, that $W$ is closed graph. Let $u_{n} \rightarrow u$ in $L^{p}(0, T ; V)$ and $x_{n} \in W\left(u_{n}\right), x_{n} \rightarrow x$ in $C([0, T] ; X)$. We claim that $x \in W(u)$. Indeed, one has

$$
x_{n}(t)=x^{*}(t)+\int_{0}^{t} \Phi(t, s)\left[f\left(s, x_{n}(s)\right)+B u_{n}(s)\right] d s .
$$

Since $f$ is a continuous function, we have $f\left(s, x_{n}(s)\right) \rightarrow f(s, x(s))$ for a.e. $s \in[0, T]$. Due to the fact that $\left\{f\left(\cdot, x_{n}(\cdot)\right)\right\}$ is $L^{p}$-integrably bounded, the Lebesgue dominated convergence theorem implies

$$
f\left(\cdot, x_{n}(\cdot)\right)-f(\cdot, x(\cdot)) \rightarrow 0 \text { in } L^{p}(0, T ; X) .
$$

In addition, since $B$ is bounded, one can assert that

$$
B u_{n}(\cdot)-B u(\cdot) \rightarrow 0 \text { in } L^{p}(0, T ; X) .
$$


Therefore, taking (3.5) into account, we arrive at

$$
x(t)=x^{*}(t)+\int_{0}^{t} \Phi(t, s)[f(s, x(s))+B u(s)] d s .
$$

The proof is completed.

Let us recall some notions which we will need in the sequel.

Definition 3.1 A subset $B$ of a metric space $Y$ is said to be contractible in $Y$ if the inclusion map $i_{B}: B \rightarrow Y$ is null-homotopic, i.e., there exists $y_{0} \in Y$ and a continuous map $h: B \times[0,1] \rightarrow Y$ such that $h(y, 0)=y$ and $h(y, 1)=y_{0}$ for every $y \in B$.

Definition 3.2 A subset $B$ of a metric space $Y$ is called an $R_{\delta}$-set if $B$ can be represented as the intersection of decreasing sequence of compact contractible sets.

A multimap $G: X \rightarrow \mathcal{P}(Y)$ is said to be an $R_{\delta}$-map if $G$ is u.s.c. and for each $x \in X$, $G(x)$ is an $R_{\delta}$-set in $Y$. Every single-valued continuous map can be seen as $R_{\delta}$-map.

The following lemma gives us a convenient condition for a set of being $R_{\delta}$.

Lemma 3.3 ([6]) Let $X$ be a metric space, E a Banach space and $g: X \rightarrow E$ a proper map, i.e., $g$ is continuous and $g^{-1}(K)$ is compact for each compact set $K \subset E$. If there exists a sequence $\left\{g_{n}\right\}$ of mappings from $X$ into $E$ such that

(1) $g_{n}$ is proper and $\left\{g_{n}\right\}$ converges to $g$ uniformly on $X$;

(2) for a given point $y_{0} \in E$ and for all $y$ in a neighborhood $\mathcal{N}\left(y_{0}\right)$ of $y_{0}$ in $E$, there exists exactly one solution $x_{n}$ of the equation $g_{n}(x)=y$.

Then $g^{-1}\left(y_{0}\right)$ is an $R_{\delta}$-set.

In order to use this Lemma, we need the following result, which is called the Lasota-Yorke Approximation Theorem (see e.g., [9]).

Lemma 3.4 Let $E$ be a normed space and $f: X \rightarrow E$ a continuous map. Then for each $\epsilon>0$, there is a locally Lipschitz map $f_{\epsilon}: X \rightarrow E$ such that:

$$
\left\|f_{\epsilon}(x)-f(x)\right\|_{E}<\epsilon
$$

for each $x \in X$.

The following theorem is the main result in this section.

Theorem 3.5 Assume the hypotheses of Theorem 2.6. Then for each $u \in L^{p}(0, T ; V), W(u)$ is an $R_{\delta}$-set.

Proof Since the nonlinearity $f$ in our problem is continuous, according to Lemma 3.4, one can take a sequence $\left\{f_{n}\right\}$ of continuous, locally Lipschitz functions such that

$$
\left\|f_{n}(t, x)-f(t, x)\right\|_{X}<\epsilon_{n} \text { for all } t \in[0, T] \text { and } x \in X,
$$

where $\epsilon_{n} \rightarrow 0$ as $n \rightarrow \infty$. Without loss of generality, we can assume that

$$
\left\|f_{n}(t, x)\right\|_{X} \leq \mu(t)\left(1+\|x\|_{X}\right)+1,
$$

for all $n$. 
Consider the equation

$$
x(t)=y^{*}(t)+\int_{0}^{t} \Phi(t, s)\left[f_{n}(s, x(s))+B u(s)\right] d s .
$$

Using the same arguments as in the previous section, one obtains the existence result for (3.6). In addition, since $f_{n}$ is locally Lipschitz, the solution of (3.6) is unique.

Let

$$
\begin{aligned}
\mathcal{G}(x) & =\left(I-\mathcal{F}^{u}\right)(x), \\
\mathcal{F}_{n}^{u}(x) & =x^{*}(t)+\int_{0}^{t} \Phi(t, s)\left[f_{n}(s, x(s))+B u(s)\right] d s, \\
\mathcal{G}_{n}(x) & =\left(I-\mathcal{F}_{n}^{u}\right)(x) .
\end{aligned}
$$

The maps $\mathcal{G}$ and $\mathcal{G}_{n}$ are proper. Indeed, we will prove this assertion, e.g., for $\mathcal{G}$. Let us show that $\mathcal{G}^{-1}(K)$ is a compact set for each compact set $K \subset C([0, T] ; X)$. Assume that

$$
\left(I-\mathcal{F}^{u}\right)(D)=K
$$

and $\left\{x_{n}\right\} \subset D$ is any sequence. Then there exists a sequence $\left\{y_{n}\right\} \subset K$ such that

$$
x_{n}-\mathcal{F}^{u}\left(x_{n}\right)=y_{n} .
$$

That is,

$$
x_{n}=x^{*}+y_{n}+\mathbf{S}\left(f_{n}+B u\right)
$$

where $f_{n}(t)=f\left(t, x_{n}(t)\right)$.

Using (F2) and the fact that $\left\{y_{n}\right\}$ is bounded in $C([0, T] ; X)$, we see that $\left\{x_{n}\right\}$ is also bounded in $C([0, T] ; X)$. Then, in turn, $\left\{f_{n}\right\}$ is bounded in $L^{p}(0, T ; X)$. Thus $\left.\left\{\mathbf{S}\left(f_{n}\right)\right)\right\}$ is equicontinuous according to Lemma 2.2 yielding the equicontinuity of the sequence $\left\{x_{n}\right\}$.

Further, taking into account (3.7) and using the fact that $\left\{y_{n}(t)\right\}$ is compact for all $t \in$ $[0, T]$, we get

$$
\chi\left(\left\{x_{n}(t)\right\}\right) \leq \chi\left(\left\{y_{n}(t)\right\}\right)+\chi\left(\left\{\mathbf{S}\left(f_{n}+B u\right)(t)\right\}\right)=0 .
$$

Thus $\left\{x_{n}\right\}$ is relatively compact and therefore $D$ is a compact set.

On the other hand, $\left\{\mathcal{G}_{n}\right\}$ converges to $\mathcal{G}$ uniformly in $C([0, T] ; X)$ and equation

$$
\mathcal{G}_{n}(x)=y
$$

has a unique solution for each $y \in C([0, T] ; X)$ as well as Eq. 3.6. Therefore, applying Lemma 3.3 we conclude that

$$
W(u)=\mathcal{G}^{-1}(0)
$$

is an $R_{\delta}$-set. The proof is completed.

\section{Invariance of the Reachability Set}

Recall some notions and facts which will be needed in the sequel.

Definition 4.1 (see, e.g., [5]). Let $Y$ be a metric space. 
(1) $Y$ is called an absolute retract (AR-space) if for any metric space $X$ and any closed $A \subset X$, every continuous function $f: A \rightarrow Y$ can be extended to a continuous function $\tilde{f}: X \rightarrow Y$.

(2) $Y$ is called an absolute neighborhood retract (ANR-space) if for any metric space $X$, any closed $A \subset X$ and continuous function $f: A \rightarrow Y$, there exists a neighborhood $U \supset A$ and a continuous extension $\tilde{f}: U \rightarrow Y$ of $f$.

Obviously, if $Y$ is AR-space then $Y$ is ANR-space.

Proposition 4.1 ([7]) Let $C$ be a convex set in a locally convex linear space $Y$. Then $C$ is an AR-space.

In particular, the last proposition yields that every Banach space is an AR-space.

The following theorem is the main tool for this section. For related results on fixed point theory for ANR-spaces, we refer the reader to [9,11,14].

Theorem 4.2 ([10, Corollary 4.3]) Let $Y$ be an AR-space. Assume that $\phi: Y \rightarrow \mathcal{P}(Y)$ can be factorized as

$$
\phi=\phi_{N} \circ \phi_{N-1} \circ \ldots \circ \phi_{1}
$$

where

$$
\phi_{i}: Y_{i-1} \rightarrow \mathcal{P}\left(Y_{i}\right), i=1, \ldots, N
$$

are $R_{\delta}$-maps and $Y_{i}, i=1, . ., N-1$ are ANR-spaces, $Y_{0}=Y_{N}=Y$ are AR-spaces. If there is a compact set $K$ such that $\phi(Y) \subset K \subset Y$ then $\phi$ has a fixed point.

We are in a position to give some hypotheses for the controllability results. The following hypothesis was introduced in [24].

(S) for each $g \in L^{p}(0, T ; X)$, there exists $v \in L^{p}(0, T ; V)$ such that

$$
\mathbf{S}_{T} B v=\mathbf{S}_{T} g,
$$

where

$$
\mathbf{S}_{T} g:=(\mathbf{S} g)(T) .
$$

It is obvious that condition (S) is fulfilled if $B$ is surjective. The following lemma was stated in [24, Lemma 2]:

Lemma 4.3 Let condition $(S)$ hold. Then there exists a continuous map $C: L^{p}(0, T ; X)$ $\rightarrow L^{p}(0, T ; V)$ such that for any $g \in L^{p}(0, T ; X)$,

$$
\begin{aligned}
& \mathbf{S}_{T} B C g+\mathbf{S}_{T} g=0, \\
& \|C g\|_{L^{p}(0, T ; V)} \leqslant \alpha\|g\|_{L^{p}(0, T ; X)}
\end{aligned}
$$

where $\alpha$ is a positive number.

Let us denote by $N_{f}$, the Nemytskii operator corresponding to the nonlinearity $f$ :

$$
\begin{aligned}
& N_{f}: C([0, T] ; X) \rightarrow L^{p}(0, T ; X), \\
& N_{f}(x)(t) *=f(t, x(t))
\end{aligned}
$$


for each $t \in[0, T]$. Obviously, $N_{f}$ is continuous. Consider the map

$$
\begin{gathered}
\mathcal{G}: \quad L^{p}(0, T ; V) \rightarrow \mathcal{P}\left(L^{p}(0, T ; V)\right), \\
\mathcal{G}(u)=C N_{f} W\left(u_{0}+u\right)
\end{gathered}
$$

where $W$ is the solution multimap and $C$ is the operator in Lemma 4.3, $u_{0} \in L^{p}(0, T ; V)$ is given. The following result is the key point in this section.

Lemma 4.4 Under assumptions $\left(\Phi_{1}\right)-\left(\Phi_{3}\right),(\mathrm{F} 1)-(\mathrm{F} 2)$ and $(S)$, there exists $r^{*}>0$ such that $\mathcal{G}$ has a fixed point in $L^{p}(0, T ; V)$ provided $\|B\|<r^{*}$.

Proof We first prove that there is a number $R>0$ such that $\mathcal{G}\left(B_{R}\right) \subset B_{R}$, where $B_{R}$ is the closed ball in $L^{p}(0, T ; V)$ centered at origin with radius $R$. Let $u \in L^{p}(0, T ; V)$ and $v \in \mathcal{G}(u)=C N_{f} W\left(u_{0}+u\right)$. Then we have

$$
\|v\|_{L^{p}(0, T ; V)} \leqslant \alpha\left\|N_{f}(\omega)\right\|_{L^{p}(0, T ; X)}
$$

for some $\omega \in W\left(u_{0}+u\right)$, where $\alpha$ is the constant given in Lemma 4.3. Hence from the definition of $N_{f}$ and assumption (F2), we deduce that

$$
\|v\|_{L^{p}(0, T ; V)} \leqslant \alpha\left(\int_{0}^{T}|\mu(s)|^{p}(1+\|\omega(s)\|)^{p} d s\right)^{\frac{1}{p}} .
$$

Now using the similar estimates as in Theorem 2.6, one gets

$$
\begin{aligned}
\|v\|_{L^{p}(0, T ; V) \leqslant} & \alpha C_{\mu}\left(1+\|\omega\|_{C([0, T] ; X)}\right) \\
\leqslant & \alpha C_{\mu}\left[1+2^{\frac{p-1}{p}}\left(C_{0}^{p}+2^{p} C_{\Phi}^{p} C_{\mu}^{p}+C_{\Phi}^{p}\left\|B\left(u_{0}+u\right)\right\|_{L^{p}(0, T ; X)}^{p}\right)^{\frac{1}{p}}\right. \\
& \left.\left(1+2^{p-1} C_{\mu}^{p} \exp \left(2^{p-1} C_{\mu}^{p}\right)\right)^{\frac{1}{p}}\right] \\
\leqslant & \alpha C_{\mu}\left[1+2^{\frac{p-1}{p}}\left(C_{0}+2 C_{\Phi} C_{\mu}+C_{\Phi}\left\|B\left(u_{0}+u\right)\right\|_{L^{p}(0, T ; X)}\right)\right. \\
& \left.\left(1+2^{\frac{p-1}{p}} C_{\mu} \exp \left(\frac{1}{p} 2^{p-1} C_{\mu}^{p}\right)\right)\right] \\
\leqslant & R_{0}+R_{1}\|B\|\left(\left\|u_{0}\right\|_{L^{p}(0, T ; V)}+\|u\|_{L^{p}(0, T ; V)}\right)
\end{aligned}
$$

where

$$
\begin{aligned}
& R_{0}=\alpha C_{\mu}\left[1+2^{\frac{p-1}{p}}\left(C_{0}+2 C_{\Phi} C_{\mu}\right)\left(1+2^{\frac{p-1}{p}} C_{\mu} \exp \left(\frac{1}{p} 2^{p-1} C_{\mu}^{p}\right)\right)\right], \\
& R_{1}=2^{\frac{p-1}{p}} \alpha C_{\mu} C_{\Phi}\left[1+2^{\frac{p-1}{p}} C_{\mu} \exp \left(\frac{1}{p} 2^{p-1} C_{\mu}^{p}\right)\right] .
\end{aligned}
$$

The last inequality tells us that if $\|B\|<\frac{1}{R_{1}}$ then there is a number $R>R_{0}+R_{1}\|B\|$ $\left\|u_{0}\right\|_{L^{p}(0, T ; V)}$ such that

$$
\|v\|_{L^{p}(0, T ; V)} \leqslant R
$$

provided $\|u\|_{L^{p}(0, T ; V)} \leqslant R$, implying $\mathcal{G}\left(B_{R}\right) \subset B_{R}$.

Finally, by the compactness of $W\left(u_{0}+B_{R}\right)$, the existence of a fixed point for $\mathcal{G}$ follows from Theorem 4.2 due to the fact that 


$$
K:=\mathcal{G}\left(B_{R}\right)=C N_{f} W\left(u_{0}+B_{R}\right) \subset B_{R}
$$

is a compact set. The proof is completed.

Consider the set

$$
\mathcal{K}_{f}:=\left\{x(T ; u): u \in L^{p}(0, T ; V)\right\},
$$

where $x(\cdot ; u)$ is a solution of (1.1) corresponding to the control $u$. This set is called the reachability set of controlled problem (1.1). Similarly, we denote by $\mathcal{K}_{0}$ the reachability set for the corresponding linear problem $(f=0)$. For the basic notions and facts of control problems, the readers are referred to $[3,4,8]$.

Definition 4.2 Problem (1.1) is said to be exactly controllable if $\mathcal{K}_{f}=X$. It is called approximately controllable if $\overline{\mathcal{K}_{f}}=X$.

We have the following assertion:

Theorem 4.5 Under the hypotheses of Lemma 4.4, we have

$$
\mathcal{K}_{f}=\mathcal{K}_{0}
$$

Proof We use the similar arguments as those in [24, Theorem 1]. Let $\xi \in \mathcal{K}_{0}$. Then there exists $u_{0} \in L^{p}(0, T ; V)$ such that

$$
\xi=x^{*}(T)+\mathbf{S}_{T} B u_{0} .
$$

Taking a fixed point $\hat{u}$ of $\mathcal{G}(\cdot)=C N_{f} W\left(u_{0}+\cdot\right)$, we set

$$
u:=u_{0}+\hat{u} \text {. }
$$

Let $\hat{x}(\cdot ; u) \in W(u)$ be a solution of (1.1) corresponding to the control $u$ such that

$$
\hat{u}=C N_{f}(\hat{x}) \text {. }
$$

Then

$$
\begin{aligned}
\hat{x}(T ; u) & =x^{*}(T)+\mathbf{S}_{T}\left(N_{f}(\hat{x})+B u\right) \\
& =x^{*}(T)+\mathbf{S}_{T} B u_{0}+\mathbf{S}_{T} N_{f}(\hat{x})+\mathbf{S}_{T} B \hat{u} \\
& =\xi+\mathbf{S}_{T} N_{f}(\hat{x})+\mathbf{S}_{T} B C N_{f}(\hat{x}) \\
& =\xi
\end{aligned}
$$

according to the definition of the operator $C$. Thus $\mathcal{K}_{0} \subset \mathcal{K}_{f}$.

Conversely, suppose that $\eta \in \mathcal{K}_{f}$. Then there exists $u \in L^{p}(0, T ; V)$ and $\hat{x} \in C([0, T] ; X)$ such that

$$
\eta=\hat{x}(T ; u)=x^{*}(T)+\mathbf{S}_{T} N_{f}(\hat{x})+\mathbf{S}_{T} B u .
$$

Denoting

$$
u_{0}=u-C N_{f}(\hat{x})
$$

we see that

$$
\begin{aligned}
x_{0}(T) & =x^{*}(T)+\mathbf{S}_{T} B u_{0} \\
& =x^{*}(T)+\mathbf{S}_{T} B u-\mathbf{S}_{T} B C N_{f}(\hat{x})
\end{aligned}
$$




$$
\begin{aligned}
& =x^{*}(T)+\mathbf{S}_{T} N_{f}(\hat{x})+\mathbf{S}_{T} B u-\left(\mathbf{S}_{T} N_{f}(\hat{x})+\mathbf{S}_{T} B C N_{f}(\hat{x})\right) \\
& =x^{*}(T)+\mathbf{S}_{T} N_{f}(\hat{x})+\mathbf{S}_{T} B u \\
& =\eta
\end{aligned}
$$

Since $x_{0}(T) \in \mathcal{K}_{0}$, one gets that $\mathcal{K}_{f} \subset \mathcal{K}_{0}$. So we have the desired conclusion.

\section{Applications to Approximate Controllability}

In this section we present two examples illustrating our result.

\section{Fractional Order Differential Control Problem}

We will consider a control system governed by a semilinear differential equation of a fractional order in a Banach space. It should be mentioned that at the present time the differential equations with fractional order have been proved to be valuable tools in the investigation of many phenomena in various fields of physics and engineering (viscoelasticity, electrochemistry, electromagnetism, etc.) and they attract the attention of many researchers (see, e.g., [1, $13,15,19,21]$ and references therein). We recall some concepts from the fractional calculus.

Let $X$ be a Banach space.

Definition 5.1 The Riemann-Liouville fractional primitive of order $\alpha>0$ of a function $f \in L^{1}(0, T ; X)$ is defined by

$$
I_{0}^{\alpha} f(t)=\frac{1}{\Gamma(\alpha)} \int_{0}^{t}(t-s)^{\alpha-1} f(s) d s,
$$

where $\Gamma$ is the Gamma function.

Definition 5.2 Let $f \in C([0, T] ; X)$. The Riemann-Liouville fractional derivative of order $\alpha \in(0,1)$ of $f$ is defined by

$$
D_{0}^{\alpha} f(t)=\frac{1}{\Gamma(1-\alpha)} \frac{d}{d t} \int_{0}^{t}(t-s)^{-\alpha} f(s) d s .
$$

Consider the problem

$$
\begin{aligned}
D_{0}^{\alpha} x(t) & =A x(t)+f(t, x(t))+\lambda u(t), \quad t \in[0, T], \\
x(0) & =0
\end{aligned}
$$

where $A$ is closed linear operator generating a compact strongly continuous semigroup $e^{t A}$ in $X, u$ takes its values in $X$ and $\lambda$ is a real parameter. The mild solution for (5.1)-(5.2) (see, e.g., $[1,19])$ is a function $x \in C([0, T] ; X)$ satisfying

$$
x(t)=\frac{1}{\Gamma(\alpha)} \int_{0}^{t}(t-s)^{\alpha-1} e^{(t-s) A}[f(s, x(s))+\lambda u(s)] d s .
$$

In this case

$$
\Phi(t, s):=(t-s)^{\alpha-1} e^{t A}
$$

satisfies $\left(\Phi_{1}\right)-\left(\Phi_{3}\right)$ with $q<\frac{1}{1-\alpha}$ (accordingly, $p>\frac{1}{\alpha}$ ). 
Let

$$
\mathcal{B} u:=\int_{0}^{T} \Phi(T, s) u(s) d s
$$

and $\mathcal{B}^{*}$ be the adjoint operator of $\mathcal{B}$. We have the following controllability result:

Theorem 5.1 Suppose that $f$ satisfies (F1)-(F2) for $p>\frac{1}{\alpha}$ and $\lambda>0$ is sufficiently small. If the implication

$$
\mathcal{B}^{*} z=0 \text { for } z \in X^{*} \Rightarrow z=0
$$

holds, then problem (5.1)-(5.2) is approximately controllable.

Proof As indicated above, if $p>\frac{1}{\alpha}$ then $\Phi$ satisfies $\left(\Phi_{1}\right)-\left(\Phi_{3}\right)$. In addition, since $B=\lambda I$ : $V=X \rightarrow X$ is surjective, the condition (S) is fulfilled. Thus the hypotheses of Theorem 4.5 are satisfied and then

$$
\mathcal{K}_{f}=\mathcal{K}_{0}
$$

The remaining arguments follow from [8, Theorem 8.8]. If (5.4) holds, we get $\overline{\mathcal{K}_{0}}=X$.

First Order Differential Control Problem with Multiple Delays

As a second example, we consider the following problem in a Banach space $X$ :

$$
\begin{aligned}
& x^{\prime}(t)=A x(t)+\sum_{i=1}^{N} A_{i} x\left(t-h_{i}\right)+f(t, x(t))+B u(t), t \in[0, T], \\
& x(\theta)=\varphi(\theta), \theta \in[-h, 0],
\end{aligned}
$$

where $h_{i} \in[0, h]$ for all $i=1,2, \ldots, N, A$ is the infinitesimal generator of a compact strongly continuous semigroup $e^{t A}$ in $X$, the operators $A_{i}: X \rightarrow X$ are bounded linear and $\varphi \in C([-h, 0] ; X)$. For the linear problem

$$
\begin{aligned}
& x^{\prime}(t)=A x(t)+\sum_{i=1}^{N} A_{i} x\left(t-h_{i}\right), t \in[0, T], \\
& x(\theta)=\varphi(\theta), \theta \in[-h, 0],
\end{aligned}
$$

we recall the definition of the fundamental solution from [27]:

Definition 5.3 The operator-valued function $\mathcal{S}(t), t \in[0, T]$ is called the fundamental solution of (5.7)-(5.8) if it satisfies

$$
\begin{aligned}
& \mathcal{S}(t)=e^{t A}+\int_{0}^{t} e^{(t-s) A}\left[\sum_{i=1}^{N} A_{i} \mathcal{S}\left(s-h_{i}\right)\right] d s, \quad t \in[0, T], \\
& \mathcal{S}(t)=0,-h \leqslant t<0, \mathcal{S}(0)=I .
\end{aligned}
$$

Following [27, Lemma 2.1], we see that $\mathcal{S}(t)$ is bounded and compact on [0,T]. Based on the notion of the fundamental solution, the mild solution of (5.5)-(5.6) is defined as

$$
\begin{aligned}
& x(t)=\mathcal{S}(t) \varphi(0)+\int_{0}^{t} \mathcal{S}(t-s)[f(s, x(s))+B u(s)] d s, t \in[0, T], \\
& x(\theta)=\varphi(\theta), \quad t \in[-h, 0] .
\end{aligned}
$$


Obviously, in this example

$$
\Phi(t, s):=\mathcal{S}(t-s)
$$

satisfies $\left(\Phi_{1}\right)-\left(\Phi_{3}\right)$ with all $1 \leqslant q \leqslant+\infty$.

Denote

$$
\begin{aligned}
\mathcal{C} & =\int_{0}^{T} \mathcal{S}(T-s) B B^{*} \mathcal{S}^{*}(T-s) d s, \\
\mathcal{R}_{\lambda} & =(\lambda I+\mathcal{C})^{-1} .
\end{aligned}
$$

We have the following assertion:

Theorem 5.2 Assume that $X$ and $V$ are Hilbert spaces. Let $f$ satisfy $(F 1)-(F 2)$ with $p=2$; $B$ be a surjective map with a sufficiently small norm $\|B\|$. If $\lambda \mathcal{R}_{\lambda} \rightarrow 0$ as $\lambda \rightarrow 0$ in strong operator topology then problem (5.7)-(5.8) is approximately controllable.

Proof Obviously, the hypotheses of Theorem 4.5 are fulfilled. So we have $\mathcal{K}_{f}=\mathcal{K}_{0}$. Therefore the conclusion is ensured by [3, Theorem 2].

\section{Conclusions}

We study the controllability problem for a system governed by a nonlinear Volterra type equation, to which the solution is not unique. Our system is derived from a wide class of evolution equations, some of which are presented in the previous section. We prove that the reachability set of our controlled problem remains invariant comparing with that of corresponding linear problem. While the existence result is ensured by the fixed point theory for condensing maps, the invariance of reachability set is proved by applying a fixed point theorem for non-convex valued multimaps, which can be factorized as a number of $R_{\delta}$-maps. This result implies that the nonlinear problem is approximately controllable, provided that the corresponding linear problem is.

Acknowledgements The work is partially supported by the Taiwan NSC - Russian FBR Grant 09-01-92003NNS. The work of V. Obukhovskii is supported by the RFBR Grant 11-01-00328; the work of N.-C. Wong is supported by the NSC Grant 99-2115-M-110-007-MY3 and the work of J.-C. Yao is supported by the NSC Grant 98-2923-E-037-001-MY3.

\section{References}

1. Agarwal, R.P., Belmekki, M., Benchohra, M.: A survey on semilinear differential equations and inclusions involving Riemann-Liouville fractional derivative. Adv. Differ. Equ. Art. ID 981728, 47 pp (2009)

2. Balachandran, K., Dauer, J.P.: Controllability of nonlinear systems in Banach spaces: a survey. J. Optim. Theory Appl. 115(1), 7-28 (2002)

3. Bashirov, A.E., Mahmudov, N.I.: On concepts of controllability for deterministic and stochastic systems. SIAM J. Control Optim. 37(6), 1808-1821 (1999)

4. Bensoussan, A., Da Prato, G., Delfour, M.C., Mitter, S.K.: Representation and Control of Infinite Dimensional Systems. Birkhaüser, Boston (2007)

5. Borsuk, K.: Theory of Retracts. Monografie Mat., vol. 44. PWN, Warszawa (1967)

6. Browder, F.E., Gupta, C.P.: Topological degree and nonlinear mappings of analytic type in Banach spaces. J. Math. Anal. Appl. 26, 390-402 (1969)

7. Dugundji, J.: An extension of Tietze's theorem. Pac. J. Math. 1, 353-367 (1951) 
8. Engel, K.-J., Nagel, R.: One-parameter semigroups for linear evolution equations. Graduate Texts in Mathematics, vol. 194. Springer-Verlag, New York (2000)

9. Górniewicz, L.: Topological Fixed Point Theory of Multivalued Mappings. 2nd edn. Springer, Dordrecht (2006)

10. Górniewicz, L., Lassonde, M.: Approximation and fixed points for compositions of $R_{\delta}$-maps. Topol. Appl. 55(3), 239-250 (1994)

11. Granas, A., Dugundji, J.: Fixed Point Theory. Springer Monographs in Mathematics. Springer-Verlag, New York (2003)

12. Kamenskii, M., Obukhovskii, V., Zecca, P.: Condensing multivalued maps and semilinear differential inclusions in Banach spaces. de Gruyter Series in Nonlinear Analysis and Applications, vol. 7. Walter de Gruyter, Berlin (2001)

13. Kilbas, A.A., Srivastava, H.M., Trujillo, J.J.: Theory and Applications of Fractional Differential Equations. North-Holland Mathematics Studies. Vol. 204, Elsevier, Amsterdam (2006)

14. Liou, Y.C., Obukhovskii, V., Yao, J.C.: Application of a coincidence index to some classes of impulsive control systems. Nonlinear Anal. 69(12), 4392-4411 (2008)

15. Machado, J.T., Kiryakova, V., Mainardi, F.: Recent history of fractional calculus. Commun. Nonlinear Sci. Numer. Simul. 16(3), 1140-1153 (2011)

16. Naito, K.: An inequality condition for approximate controllability of semilinear control system. J. Math. Anal. Appl. 138(1), 129-136 (1989)

17. Naito, K.: On controllability for a nonlinear Volterra equation. Nonlinear Anal. 18(1), 99-108 (1992)

18. Naito, K., Park, J.Y.: Approximate controllability for trajectories of a delay Volterra control system. J. Optim. Theory Appl. 61(2), 271-279 (1989)

19. Obukhovskii, V., Yao, J.-C.: Some existence results for fractional functional differential equations. Fixed Point Theory 11(1), 85-96 (2010)

20. Obukhovskii, V., Zecca, P.: Controllability for systems governed by semilinear differential inclusions in a Banach space with a noncompact semigroup. Nonlinear Anal. 70(9), 3424-3436 (2009)

21. Podlubny, I.: Fractional differential equations. An introduction to fractional derivatives, fractional differential equations, to methods of their solution and some of their applications. Mathematics in Science and Engineering, vol. 198. Academic Press, San Diego (1999)

22. Qin, Y.: Nonlinear parabolic-hyperbolic coupled systems and their attractors. Operator Theory: Advances and Applications, vol. 184. Advances in Partial Differential Equations (Basel). Birkhaüser Verlag, Basel (2008)

23. Quinn, M.D., Carmichael, N.: An approach to nonlinear control problems using fixed-point methods, degree theory and pseudo-inverses. Numer. Funct. Anal. Optim. 7(23), 197-219 (1984/1985)

24. Seidman, T.I.: Invariance of the reachable set under nonlinear perturbations. SIAM J. Control Optim. 25(5), 1173-1191 (1987)

25. Triggiani, R.: A note on the lack of exact controllability for mild solutions in Banach spaces. SIAM J. Control Optim. 15(3), 407-411 (1977)

26. Triggiani, R.: Addendum: "A note on the lack of exact controllability for mild solutions in Banach spaces". SIAM J. Control Optim. 18(1), 98-99 (1980)

27. Wang, L.W.: Approximate controllability for integrodifferential equations with multiple delays. J. Optim. Theory. Appl. 143(1), 185-206 (2009) 